\title{
LA HOZ DE LA EDAD DEL BRONCE DEL «MAS DE MENENTE» (ALCOI, ALACANT). APROXIMACIÓN A SU TECNOLOGÍA Y CONTEXTO CULTURAL
}

\author{
JOAQUÍN JUAN CABANILLES \\ Servicio de Investigación Prehistórica. Valencia
}

\begin{abstract}
La hoz del Mas de Menente constituye, dentro del marco del Mediterráneo occidental europeo, uno de los pocos ejemplares de este tipo de herramientas prehistóricas que es posible reconstruir gracias a haberse conservado parte de su mango en madera. El estudio de este mango, así como de las probables armaduras constitutivas, permite un acercamiento más directo a la tecnología de la hoz en las últimas etapas de la Prehistoria reciente en el ámbito peninsular, concretamente a aquellas que corresponden al Bronce Valenciano. Para entender el caso de Menente en su justo contexto crono-cultural, ha sido necesario pasar revista a la evolución de las hoces en el mismo ámbito.
\end{abstract}

The sickle of Mas de Menente preserves the handle of wood and can be reconstructed. The analysis of the handle, and of the probable frames, makes easy the knowledge of the technology of the sickle in the last stages of the Peninsular Prehistory, specially of the Valencian Bronze. For it we have revised the evolution of the sickles in this area.

El poblado prehistórico del Mas de Menente, claramente encuadrado en la cultura del Bronce Valenciano, fue uno de los primeros asentamientos de esta índole excavados por el Servicio de Investigación Prehistórica de la Diputación Provincial de Valencia (BALLESTER, 1929). Con anterioridad a los trabajos del S.I.P., el yacimiento había sido dado a conocer por su descubridor y primer prospector F. Ponsell (1926), siendo ya fruto de las investigaciones llevadas a cabo por aquella institución la definitiva publicación del mismo a cargo de L. Pericot y el propio Ponsell (PERICOT Y PONSELL, 1929).

Entre otros aspectos, la importancia del Mas de Menente radica en haber permitido recuperar en excavación los restos de un mango de madera correspondiente a una hoz prehistórica, constituyen- do uno de los pocos hallazgos en este sentido que pueden contabilizarse en el ámbito del Mediterráneo occidental europeo.

La significación de este hallazgo se ve acrecentada más aún por tratarse de uno de los pocos soportes de hoz que actualmente se conserva en el mismo ámbito. Prácticamente sería el único conservado, si nos atenemos a una reciente síntesis sobre las hoces preshistóricas de la cuenca mediterránea (CAMPS-FABRER Y COURTIN, 1982-83) y si dejamos de lado, por quedar fuera del marco geográfico-cultural estrictamente mediterráneo, los vestigios de esta naturaleza documentados en los poblados palafíticos del Norte de Italia y Suiza. No obstante, y por lo que hace a esta última consideración, no habría que olvidar otro caso célebre de hoz también encontrada entera en su día, de 
igual manera en el marco peninsular, pero desgraciadamente desaparecida en la actualidad: el ejemplar de la Cueva de los Murciélagos de Albuñol (Granada) (GONGORA, 1868; citado además en VAYSON DE PRADENNE, 1918-19; CURWEN, 1930; MONTEAGUDO, 1956; CAMPS-FABRER Y COURTIN, 1982-83). Asimismo, y toda vez que no se trata de un hallazgo real sino de una reproducción, señalaríamos el armazón de hoz del Acebuchal (Carmona, Sevilla), construido y experimentado de antiguo por G. Bonsor (citado en VAYSON DE PRADENNE, 1918-19; CURWEN, 1930; MONTEAGUDO, 1956); de este mango reconstruido tampoco se tiene noticia de su conservación en el momento presente.

Desde su descubrimiento y publicación, la hoz del Mas de Menente ha sido cita obligada en la mayor parte de la bibliografía relacionada con la Cultura del Bronce Valenciano. Ultra su utilización como elemento comparativo, la pieza ha devenido un excelente modelo a la hora de ilustrar la orientación tecnológica de la industria lítica en sílex de la etapa. De este modo, su representación bien en dibujo o fotografía no ha faltado en importantes trabajos de sintesis (TARRADELL, 1965: 48-49, foto n. ${ }^{\circ}$ 10; APARICIO, 1976: 14L8, fig. 13, A; ENGUIX, 1980: 168, foto, abajo; MARTÍ, 1983: 96, fig. 29), en algunos catálogos de materiales (FLETCHER, 1974: 104, foto, abajo), e incluso en algunas enciclopedias generales (G.E.R.V., 1973: 261, foto).

A pesar de ello, esta pieza singular apenas ha sido objeto de un estudio detenido. En las reseñas originales sobre el Mas de Menente (PONSELL, 1926; PERICOT Y PONSELL, 1929), y en lo que respecta a la valoración de los materiales exhumados, el propio espacio dedicado a la hoz es bastante exiguo, quedando reducida la información a consignar algunos datos aislados sobre las dimensiones generales del mango y de las armaduras integrantes, sin entrar en más detalles técnicos, mucho menos en una valoración contextual del útil. Por otra parte, y entre la bibliografía especializada consagrada en algún modo al tema de las hoces prehistóricas, únicamente hemos encontrado referencias a la hoz de Menente en dos trabajos por lo demás bastante dispares. El primero de ellos, firmado por V. G. Childe, está centrado en el estudio de las hoces balanceadas (CHILDE, s/r), y las referencias a la pieza de Menente radican en enumerar - brevemente - las características que permiten su inclusión en este tipo de hoces, cuestión so- bre la que más adelante volveremos, dado que esta categorización se apoya en una morfología del mango que no concuerda con lo que realmente puede observarse. El segundo trabajo es la conocida sintesis de L. Monteagudo concerniente a esta clase de herramientas (MONTEAGUDO, 1956). Dicho autor se limita a recoger directamente los datos proporcionados por Pericot y Ponsell, utilizando el ejemplar de Menente para ilustrar uno de sus tipos específicos de hoz (Ibid, fig. 1, n. ${ }^{\circ} 13$ ). La novedad reside en que Monteagudo sí intenta un enmarque tecno-cultural de la hoz, empresa que no se había llevado a cabo hasta el momento.

En suma, estos serían los únicos tratamientos que ha recibido la hoz de Menente. Así, pues, dado su innegable interés, creemos que se hace necesario el reestudio de la pieza desde unas bases analíticas

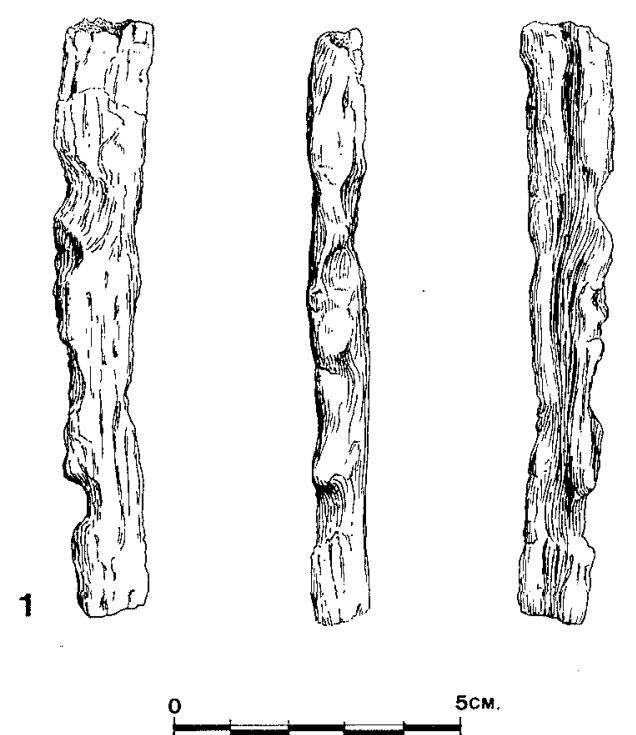

2
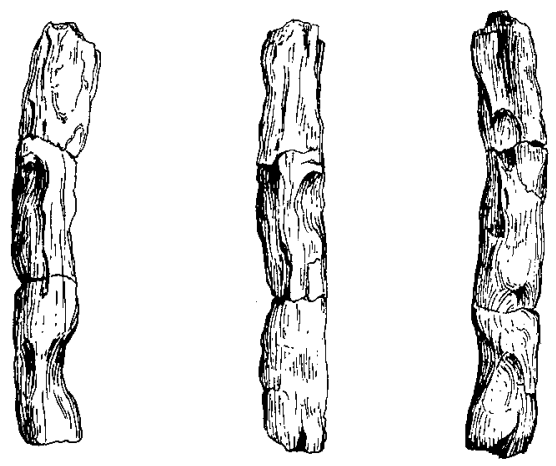

Fig. 1.-Fragmentos del mango de la hoz de Menente. (Dibujo de F. Chiner). 
más expresas, a fin de conseguir un acercamiento directo a la realidad tecnológica de las últimas etapas de la Prehistoria reciente en nuestro ámbito de trabajo.

En este propósito se inscribe el presente artículo. Para ello hemos dedicado un primer capítulo al estudio concreto de la hoz, atendiendo a las características del mango conservado y a la de las posibles armaduras constitutivas; en segundo lugar, trataremos de valorar el útil en su adecuado marco tecnológico y cultural.

\section{ESTUDIO DE LA HOZ}

Antes de pasar al estudio analítico propiamente dicho, conviene indicar que tanto el mango como algunas de las armaduras que pudieron formar
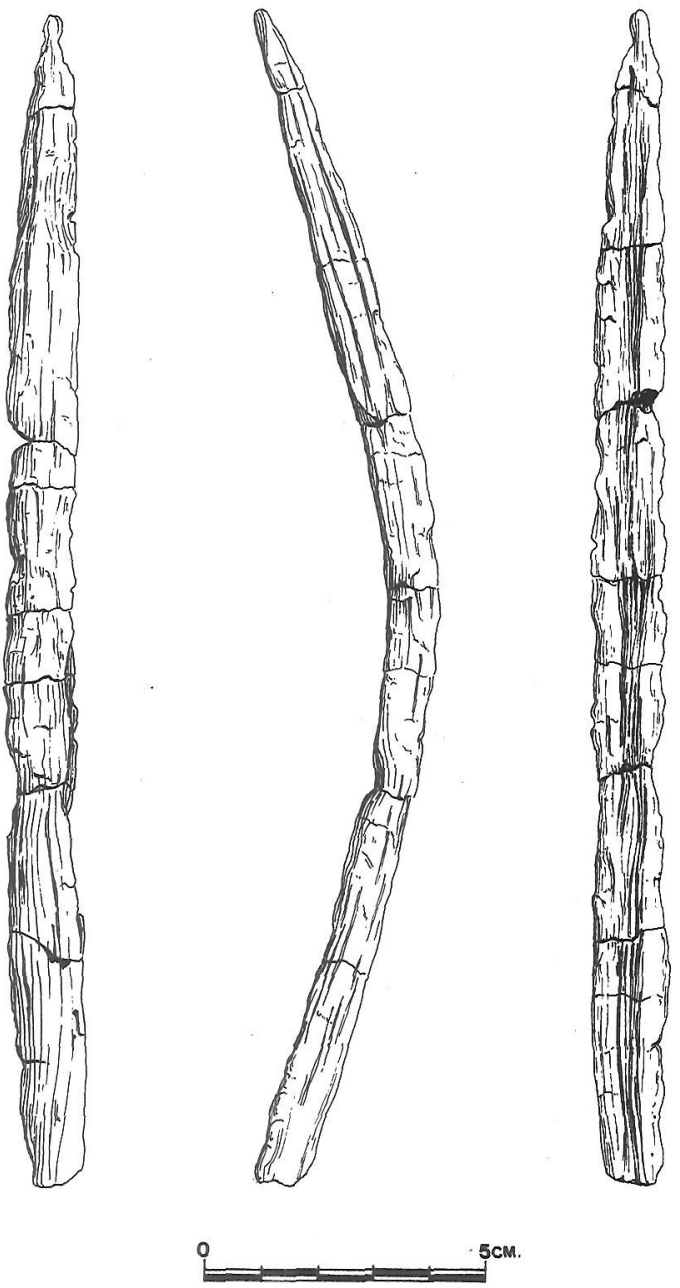

Fig. 2.-Fragmento del mango de la hoz de Menente. (Dibujo de F. Chiner). parte de la hoz de Menente se encuentran depositadas en el Museo del Servicio de Investigación Prehistórica de la Diputación Provincial de Valencia, junto a los restantes materiales procedentes de la excavación de este yacimiento.

\subsection{El mango}

Del mango de la hoz de Menente se conservan en la actualidad tres fragmentos altamente deteriorados (figs. 1 y 2 ; lám. I). Se tiene pocas precisiones sobre las circunstancias del hallazgo; únicamente relataba el propio Ponsell que «la madera que constituía la hoz» habría subsistido «probablemente por haber estado protegida por una gran piedra que tenía encima» (PONSELL, 1926: 6). Por referencias orales del director del S.I.P., E. $\mathrm{Pla}$, sabemos que el mango se encontró completa-

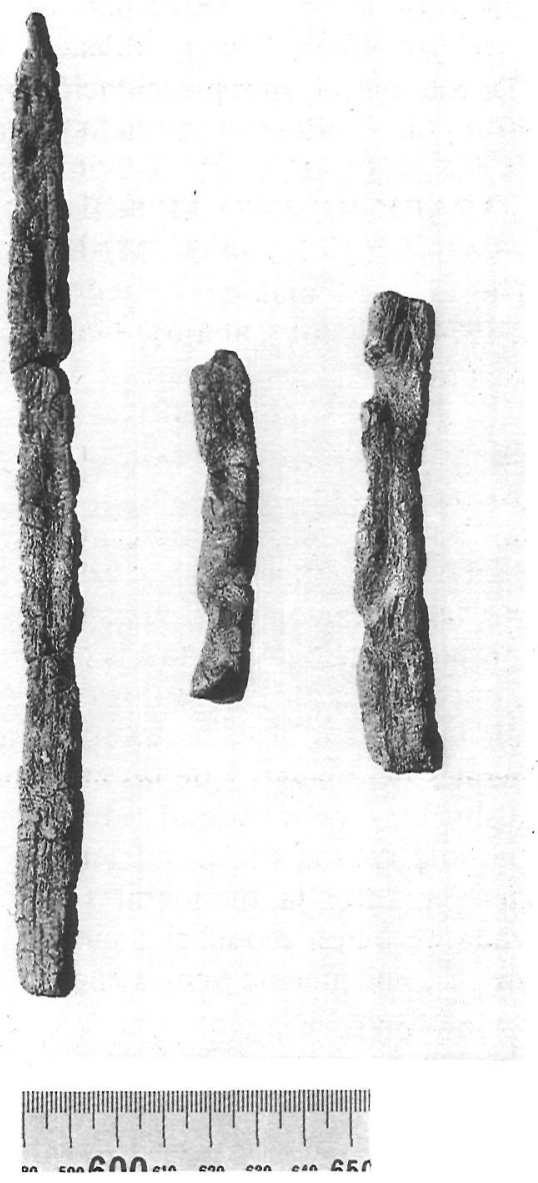

Lám. I.-Vista de la cara interna de los fragmentos del mango de la hoz de Menente. 
mente astillado y ya muy alterado; trasladado al laboratorio de esta entidad, se procedió a su consolidación y reconstitución, lo que dejó el montante reducido a las tres porciones conocidas. De esta forma definitiva, y con la conexión ficticia de las tres partes (sin una fijación real), es como ya aparece representada la hoz (fig. 3, n $^{\circ} 1$ y 3 ) en las distintas ilustraciones de que ha sido objeto, normalmente mediante fotografía, y manteniendo siempre la forma modélica que primeramente presentara Ponsell (1926, lám. VI-A).

Tal como hemos apuntado, el estado de conservación de los distintos fragmentos es bastante precario; cosa nada extraña si se tiene en cuenta las pocas posibilidades de preservación que ofrece la madera fuera de ciertos medios apropiados, en el que el caso del Mas de Menente puede considerarse como de excepcional. Ello plantea toda una serie de problemas a la hora de un estudio morfoanalítico y tecnológico, pues es evidente que se verán afectadas en su objetividad real las diferentes valoraciones métricas y descriptivas que podamos realizar; con estas limitaciones habrá que contar en el momento de la necesaria interpretación de los datos.

Para una mejor apreciación de detalles, hemos creído conveniente efectuar el análisis descriptivo de las tres partes del mango por separado. Posteriormente haremos la valoración de conjunto.

Fragmento a (Fig. 1, n. ${ }^{\circ}$ 1; lám. I)

Desde el primer montaje de la hoz - como ya hemos indicado el mismo que siempre ha prevalecido-, esta porción aparece constantemente en disposición proximal (fig. $3, \mathrm{n}^{\circ} 1$ ), pareciendo querer asignársele el papel de mango en sentido estricto o empuñadura.

Dimensionalmente, presenta una longitud de $10,48 \mathrm{~cm}$., una anchura que varía de 1,05 a 1,67 $\mathrm{cm}$. (valores mínimo y máximo respectivamente), y un espesor comprendido entre 1,05 y $1,14 \mathrm{~cm}$. La sección es de tendencia semicircular: la parte redondeada correspondería, lógicamente, al dorso del mango y la superficie plana a su cara interna o espacio funcional, donde irían dispuestas las armaduras conformadoras del filo cortante. Para este fin, puede observarse en dicha cara interna las marcas de la ranura de fijación, cuya anchura varía de 0,32 a $0,73 \mathrm{~cm}$., aproximadamente, sin llegar en ningún trecho a los $2 \mathrm{~mm}$. de profundidad; estas medidas son totalmente referenciales dada la per- ceptible y repetidamente señalada alteración estructural de éste y los demás restos de mango. Indicaremos que la ranura, aunque difuminada en algunos tramos, puede seguirse en toda la extensión de la cara interna del fragmento (lám. I).

Volviendo de nuevo a ello, estructuralmente el fragmento $a$, al igual que los restantes, manifiesta una fuerte pérdida de materia; sintomático de esto son las numerosas descamaciones y vaciados que le dan una textura rugosa y desigual, afectando obviamente a sus proporciones y aspecto real. Por otro lado, hay que señalar el ligero combamiento lateral que presenta, manteniéndose, en cambio, prácticamente rectilíneo según el plano funcional. Otra particularidad significativa a destacar es el aparente aserrado que of rece una de sus extremidades, concretamente la más estrecha, aquella que en algunas ocasiones se ha representado como el extremo proximal de la hoz (TARRADELL, 1965; APARICIO, 1976). Indudablmente, este carácter habrá sido el sugeridor de tal disposición y, consiguientemente, de la funcionalidad como mango de este fragmento, lo que será discutido en otro momento del presente artículo.
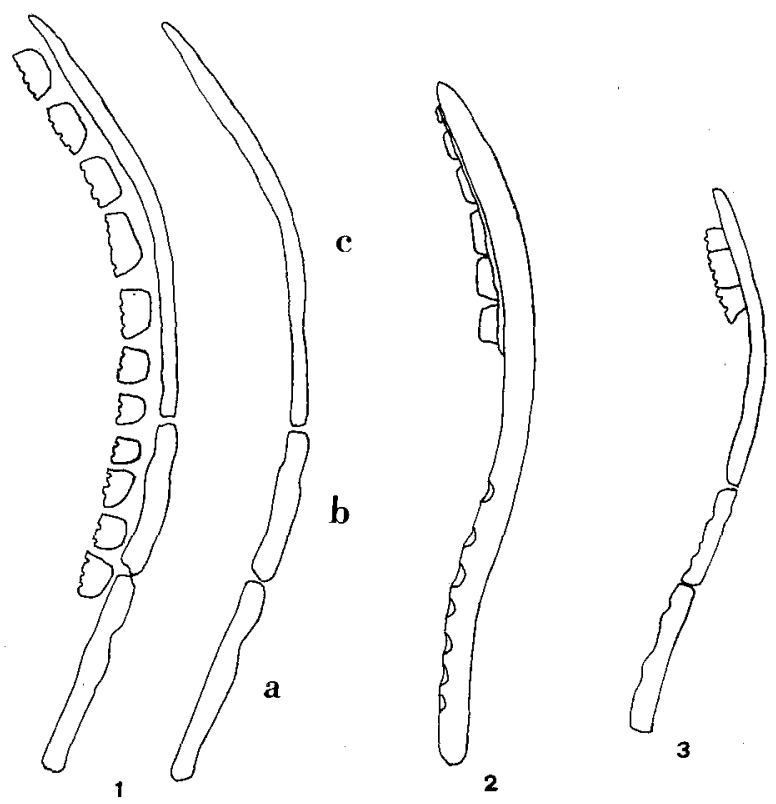

Fig. 3.-Reconstrucciones y montajes de la hoz de Menente: N. ${ }^{\circ}$ 1, según M. Tarradell (1965); n. ${ }^{\circ}$ 2, según V. G. Childe (s/r); n. ${ }^{\circ} 3$, según F. Ponsell (1926). Los n. ${ }^{\circ} 1$ y 3 son dibujos sobre foto. 
Fragmento $b$ (Fig. 1, n. ${ }^{\circ}$ 2, lám. I)

En el montaje clásico, ocupa la posición medial de la hoz (fig. 3, n. $^{\circ} 1$ ), siendo el menor de los fragmentos constitutivos.

Tiene una longitud de 7,65 $\mathrm{cm}$., una anchura comprendida entre 1.01 y $1.32 \mathrm{~cm}$., y un espesor que va de 1 a $1,31 \mathrm{~cm}$. (siempre valores mínimos y máximos). La sección es de tendencia trapezoidal, bastante irregular.

Es el fragmento peor conservado, con un fuerte grado de alteración y deformación. Esto llega a impedir diferenciar con exactitud el dorso de la cara interna, máxime cuando también son difíciles de reconocer las huellas de la ranura de fijación. De todos modos, basándonos en una serie de estrias terminales, que bien pudieran corresponder a dicha entalladura, hemos orientado el fragmento de la forma en que aparece en la figura $1\left(n .^{\circ} 2\right)$; ver también lám. $I$.

\section{Fragmento c (Fig. 2; lám. I)}

Corresponde sin dudas a la extremidad distal del mango de la hoz (fig. $3, \mathrm{n} .^{\circ} 1$ ), ofreciendo una marcada curvatura y siendo el mayor de los fragmentos conservados.

La longitud de la cuerda del arco que forma, es de $20,90 \mathrm{~cm}$., mientras que el arco mide $22,10 \mathrm{~cm}$.; la anchura ofrece valores entre 1,42 y $1,03 \mathrm{~cm}$. y el espesor varía de 1,01 a $0,72 \mathrm{~cm}$. La sección es nuevamente de tendencia semicircular.

Los vestigios de la ranura son bien visibles en la cara interna del fragmento (lám. I), presentando anchuras que oscilan entre 0,35 y $0,64 \mathrm{~cm}$., y alcanzando en algún punto una profundidad máxima de $2 \mathrm{~mm}$. El surco, en su localización, puede seguirse en prácticamente toda la extensión del fragmento, deteniéndose a unos escasos $2,5 \mathrm{~cm}$. del extremo distal, el cual se presenta a su vez levemente apuntado. Estos caracteres, juntamente con la disminución progresiva de la anchura del fragmento en esa misma dirección, verifican su atribución como parte terminal de la hoz.

Tras las primeras consideraciones efectuadas y el análisis individual de cada fragmento, las características morfológicas, métricas y de conservación actuales del mango de la hoz de Menente quedarían así resumidas:

- Se trata de los restos de un mango de hoz fraccionado en tres porciones. Estas son el resultado de una consolidación posterior, puesto que en el momento del hallazgo la desmembración de la pieza sería casi completa, lo que viene revelado, entre otras cosas, por las numerosas líneas de fractura que presentan cada uno de los fragmentos. A subrayar que las tres porciones son inconexas entre sí.

- El grado de alteración de los fragmentos es elevado, manifestando una acusada pérdida de materia y una gran degradación estructural.

- Unidas las tres porciones, el mango se presenta - visto en perfil- sensiblemente curvado, con un dorso redondeado y una cara interna perceptiblemente aplanada, en la cual pueden reconocerse las trazas de la ranura de fijación para las armaduras cortantes. Los restos de la ranura, bastante indeterminados en el fragmento teóricamente medial, pueden seguirse claramente, y en prácticamente toda su extensión, en las restantes porciones del mango. El fragmento distal, cuya anchura disminuye progresivamente, acaba en un leve apuntamiento, lo que confirma su posición terminal en la hoz.

- Tomadas en su conjunto, las mediciones efectuadas proporcionan las siguientes dimensiones totales para el mango:

Longitud aproximada (según la long. de la cuerda del arco para el fragmento c): 39,03 $\mathrm{cm}$.

Longitud aproximada (según la long. del arco para el mismo fragmento $\mathrm{c}$ ): $40,23 \mathrm{~cm}$.

Anchura media: $1,26 \mathrm{~cm}$.

Espesor medio: $1,01 \mathrm{~cm}$.

Las medias de anchura y espesor se han sacado sobre un total de 22 mediciones para cada una de las dimensiones, escalonadas regularmente a todo lo largo del montante. serían:

- Las dimensiones aproximativas de la ranura

Anchura media: $0,47 \mathrm{~cm}$.

Profundidad máxima: $2 \mathrm{~mm}$.

\subsection{Las armaduras}

La excavación del Mas de Menente proporcionó, además de los restos del mango estudiado, una gran cantidad de elementos dentados en sílex que, aunque con seguridad pertenecen al tipo de piezas que armarían la hoz, no fueron encontrados en asociación directa con el montante; este hecho conviene recalcarlo por lo que puede llevar a confusión la vista de las reconstituciones originarias de 
la hoz, en que siempre aparece un número determinado de estas armaduras, en algunas ocasiones variante, formando parte de ella. En ello mismo insistía Monteagudo cuando subrayaba, refiriéndose al caso de Menente, que «la hoz de madera» se habría conservado «al parecer, separada de los subrectángulos dentados, aunque la foto - se refiere a la que ilustra las publicaciones primeras de Ponsell (1926) y Pericot y Ponsell (1929) - muestra tres de estos atados a la hoz». (MONTEAGUDO, 1956: 496). Unicamente avanzaremos ahora que el tema de la relación mango/armaduras será tratado con más detenimiento en el punto siguiente, dedicado a la hipotética reconstrucción de la hoz.

Por lo que concierne a este epígrafe, es interesante recordar el preciso comentario realizado por Pericot y Ponsell sobre las armaduras recuperadas en el Mas de Menente, en el cual se señalaban algunas de sus características más notables:

«Las hojas de sierra son en número de 68 ; las hojas de sílex se han preparado de manera a dejar un lado grueso, el lado que sirve para enmangar en una lámina de madera, y otro lado aguzado, en el que se tallan los dientes de la sierra; así es que tales hojas vienen a tener una sección análoga a la de nuestras navajas de afeitar. La longitud de estas pequeñas hojas de sierra varía desde 1,7 a 3,3 cms, su anchura de 1 a $1,6 \mathrm{cms}$ y el grueso suele ser poco mayor de medio centímetro. Es curioso que todas ellas (algunas no tienen más que un diente) están muy desgastadas y los dientes muy poco salientes ya, mientras la anchura de estos llega hasta $0,5 \mathrm{cms}$. Seis de estas hojas aparecieron en la habitación $n .^{\circ}$ IV, junto a los restos de hoz de madera de que hablaremos, por lo que cabe suponer formarían parte de ésta». (PERICOT Y PONSELL, 1929: 5-6).

Habría que aclarar de nuevo que, aunque aparecieron en el mismo departamento que el mango, dato a tener en cuenta, las seis armaduras de que hablan Pericot y Ponsell no se encontraban asociadas directamente a éste.

También hay que apuntar escuetamente que las «hojas de sierra» que mencionan estos autores corresponden al tipo diente de hoz, denominación que, con posterioridad, ha devenido más usual en la bibliografía del Bronce Valenciano. En esta etapa cultural es donde estas piezas alcanzan mayor generalización, llegando a ser consideradas por al- gunos autores como verdaderos fósiles guía, caracterizando morfológicamente, casi por exclusión, la producción lítica de la fase.

El diente de hoz ha sido definido por $\mathrm{J}$. Fortea como «pieza corta generalmente sobre lámina, con extremidades preparadas bien por fractura simple, bien retocada mostrando una denticulación muy regular en un borde, obtenida por muescas simples directas, inversas o bifaces») (FORTEA, 1973: 107). Formalizados tipológicamente, los dientes de hoz han sido asimismo justamente contextualizados por Martí, al haber marcado las claras distancias morfológicas y cronológico-culturales que las separan de los «elementos de hoz» neolíticos (MARTÍ, 1977: 89-90).

No obstante, estas piezas prácticamente no han sido estudiadas desde un plano estrictamente tecnológico. Las carencias en ese sentido son bien patentes en los trabajos habituales. Por esto mismo, intentaremos una aproximación a la tecnología - tanto básica como específica - de los dientes de hoz tomando como ejemplo el conjunto suministrado por el Mas de Menente. Es obvio que el análisis de esta concreta colección sólo puede proporcionar -en principio- unos resultados parciales, indicadores únicamente de los modos de fábrica específicos al grupo de Menente; ello así expuesto con el ánimo de no entrar en valoraciones apriorísticas generalizadoras, hasta que no se haya realizado el pertinente estudio de conjunto - en todas sus facetas- de los dientes de hoz del Bronce Valenciao, para cuyo proyecto la tentativa aquí expuesta puede constituir un primer punto de partida. Evidentemente, un estudio de esta índole pasaría por la contrastación, una vez analizadas, de las distintas series proporcionadas por los yacimientos conocidos, y habría de enmarcarse en el estudio más general de la industria lítica de la etapa, prácticamente sin tratamiento adecuado aún en el momento actual.

Por lo demás, el análisis particular de los dientes de hoz de Menente tiene en sí mismo un claro significado, en la medida que puede aportar un conocimiento más efectivo de las características esenciales de las armaduras integrantes de la hoz en consideración.

Centrándonos ya en ello, y en cuanto a la materia prima, los dientes de hoz de este yacimiento se hallan elaborados exclusivamente en sílex. Se trata de un sílex de bastante buena calidad, de grano medio, normalmente opaco, y con una gama de colo- 
res en que son predominantes el marrón y el gris, con diversas tonalidades, preferentemente oscuras.

Por lo que respecta a la tecnología básica, es bastante difícil dilucidar el tipo de soporte empleado para la ulterior conformación de estas piezas, sobre todo cuando no se tiene información directa sobre la clase de núcleos ni de los restos y productos de talla en bruto. Parece ser que en Menente se utiliza una talla indiferenciada, aprovechándose tanto productos laminares, de sección más o menos gruesa, como lascas de características similares. En algunos útiles acabados son visibles las aristas dorsales de extracción, pero es también frecuente que no las presenten, lo que aumenta la dificultad de reconocimiento del soporte; el que éste sea sobre lasca se ha podido verificar en ocasiones a partir de las ondas de percusión observables en la cara ventral, cuya disposición hacía improbable una extracción laminar (fig. $4, n .^{\circ} 3$ y 8); asimismo hay que hacer constar la irregularidad de las aristas dorsales cuando éstas aparecen, lo que viene a incidir en la idea de una talla no demasiado especializada, en que prácticamente se aprovecha todo tipo de producto resultante con tal que se adapte a las necesidades requeridas.

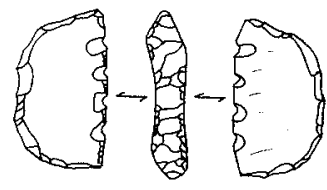

1

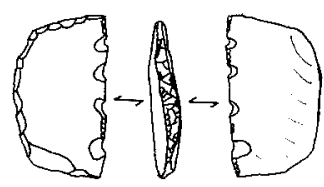

3

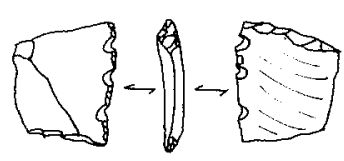

5

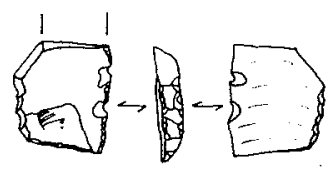

7

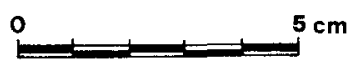

Fig. 4.-Dientes de hoz del Mas de Menente.
En su aspecto morfológico general, los dientes de hoz de Menente presentan contornos de diversas modalidades, bien rectangulares o subrectangulares (fig. 4, n. $^{\circ} 2$ ), bien subtrapezoidales -dominantes-(fig. $4, n .^{\circ} 4$ ), o de tendencias semicirculares (fig. $4, n .^{\circ} 1$ ); esto estaría en relación con la definitiva conformación de las piezas, cuyas características técnicas específicas pasamos a detallar. Generalmente, las armaduras examinadas ofrecen uno de los bordes (o dorso en su sentido de oposición al filo) bien abatido, con un retoque abrupto normalmente vertical, de técnica bipolar - caso más frecuente-, también directa y, en menor medida, de dirección inversa (fig. 4, n. ${ }^{\circ} 8$ ); en algunos casos, el dorso suele presentar una ancha superficie, por haber estado realizado sobre la parte más espesa del soporte: a la altura de la arista central, llegando a veces a eliminarla, en el caso de productos laminares, o aprovechando la extremidad proximal (cerca del talón o el bulbo) o plataformas laterales en el caso de las lascas; esto hace que disminuya progresivamente el espesor del útil en dirección al filo, lo que viene a recordar la «sección análoga a nuestras navajas de afeitar» que señalaban Pericot y Ponsell.

Los lados cortos o extremos de estas armaduras aparecen casi siempre truncados, con las mismas técnicas que las observadas para el dorso, a menudo conjugadas sobre una misma pieza. Así, pueden detectarse bilateralmente truncaduras verticales por retoque abrupto bipolar (fig. $4, n .^{\circ} 1$ ), por retoque abrupto directo (fig. $4, \mathrm{n}^{\circ} 6$ ), alterno (directo-inverso) (fig. $4, \mathrm{n},{ }^{\circ} 5$ ), u oponiéndose un retoque directo - casi nunca inverso - a otro bipolar (fig. $4, n .^{\circ} 4$ ). También puede encontrarse algún lado corto sin retoque, aunque fracturado con toda seguridad intencionalmente; la fractura recotada, en estos casos, suele ser única, combinándose en el otro extremo con un retoque de cualquiera de los modos señalados (fig. $4, n .^{\circ} 7$ y 8 ). La unión del lado corto con el dorso puede no ser totalmente angular, constituyendo en ocasiones el primero una mera prolongación del segundo, cuando se trata de dorsos más o menos curvos, lo que da a la pieza una apariencia de un ancho y tosco segmento de círculo, siendo normal que se prolongue a su vez el mismo tiempo de retoque, indistintamente por uno o ambos lados (fig. 4, n. ${ }^{\circ} 1$ y 3 ).

El filo o parte activa, naturalmente el borde opuesto al dorso, presenta los característicos dientes o resaltes definitorios de este tipo de armadu- 
ras. Dichos dientes, apenas destacados y conservando siempre la delineación primaria del filo bruto, vienen determinados por una serie de pequeñas muescas espaciadas, nunca contiguas. Generalmente, las muescas han sido producidas por presiones bifaciales, denotando su intencionalidad, y su amplitud puede variar de 0,7 a cerca de $3 \mathrm{~mm}$. La distancia entre muescas, sujeta a variación según el tamaño y número de dientes del filo, suele oscilar entre 3 y $6 \mathrm{~mm}$. de media, de acuerdo con la señalada relación particular dientes/filo de cada pieza; ello hace que dentro de cada una de ellas esta distancia se muestre relativamente homogénea, dando al fílo - conforme apuntábamos al principio - una lineación y una forma general bastante regular. El número de muescas, normalmente también sujeto a las dimensiones del filo y por extensión al propio tamaño de la pieza, puede ir de 2 a 4 en las armaduras completas, siendo más frecuentes las series de 3 . En los dientes o porción del filo entre muescas es corriente observar, alcanzando toda o casi toda su extensión, pequeños retoques más o menos contínuos que deben tener - evidentemente - un origen funcional; su modo es simple o de tendencia plana y su dirección variable, indistintamente directa, inversa, alternante o bifaz, combinándose todas ellas en algún caso; lo más usual es que estos pequeños retoques se desarrollen más intensamente en la parte del fillo de una sola de las caras de la pieza, sin una preferencia notable: bien la dorsal, bien la ventral. La pátina brillante, significativa del empleo de estos útiles en faenas agrícolas o en el simple tallado de hierbas, no siempre es perceptible a simple vista; en las piezas en que puede apreciarse (fig. $4, \mathrm{n} .^{\circ} 2$ ), el lustre alcanza poca extensión facial, ubicándose marginalmente en los tramos correspondientes a los dientes de la armadura, siempre en disposición paralela respecto al eje del útil; esto aboga por una colocación lineal de las piezas formando un fílo contínuo en la hoz.

En cuanto a sus dimensiones, y en las piezas examinadas, los dientes de hoz de Menente presentan longitudes comprendidas entre 1,56 y $2,80 \mathrm{~cm}$.; la anchura puede variar de 1,46 a $1,79 \mathrm{~cm}$. y el espesor de 0,20 a $0,55 \mathrm{~cm}$.

Haciendo abstracción de lo expuesto, las características técnicas generales de los dientes de hoz del Mas de Menete quedarían así resumidas:

- El sílex como materia prima exclusiva.
- Soportes tanto en hojas como en lascas (talla indiferenciada).

- Siluetas subtrapezoidales -dominantes-, también subrectangulares y de tendencias semicirculares -éstas en menor proporción- como resultado de la conformación especifica.

- El retoque abrupto, normalmente vertical, de técnica bipolar, directa o inversa para la definición de dorsos y truncaduras. Poca presencia de bordes-dorso y lados cortos no retocados.

- Filos denticulados por muescas regularmente espaciadas, obtenidas por presión bifacial y generalmente en número de tres.

- Lustre más o menos intenso, no siempre perceptible, y en disposición paralela respecto al eje de la armadura, extendiéndose por todo lo largo del filo.

- Unas dimensiones medias centradas, para la longitud, en $2,30 \mathrm{~cm}$.; para la anchura, en 1,61 cm. y para el espesor, en $0,38 \mathrm{~cm}$.

\subsection{Ensayo de reconstrucción}

Vistas las características de las armaduras y especialmente del mango, un primer problema que se plantea para la reconstrucción de la hoz de Menente es el de si en realidad los restos conservados corresponden a una pieza entera o si son solamente una parte de ella.

Por la forma en que habitualmente se ha representado, dejando un espacio proximal libre de dientes y que parece querer indicar el lugar de la empuñadura, podría inferirse que siempre ha prevalecido, a la hora de su montaje, la idea de un hoz completa; esta presunción se ve llevada al máximo en la reconstrucción efectuada por Childe (fig. 3, n. ${ }^{\circ}$ ).

Sin embargo, hay varios aspectos que hacen pensar lo contrario. El primero y principal sería la presencia de los restos de la ranura de fijación en los tres fragmentos conservados, pudiéndose seguir con más o menos fuerza en toda la extesión de la cara interna de los mismos; podría objetarse el caso del fragmento $b$, donde apenas son visibles las marcas del surco, pero su situación medial en la hoz (al menos entre los fragmentos $a$ y $c$ ) es del todo incuestionable. El segundo carácter sería la evidente línea de fractura que presente el fragmento a 
en su extremo teóricamente más proximal, lo que en buen sentido nos lleva a suponer que se continuaría en esa dirección.

Para aclarar estos puntos tendríamos que ver en que nos hemos basado para orientar y emplazar los fragmentos dentro de la disposición general de la hoz -ver fig. 5-. En primer lugar habría un motivo lógico, relativo a las características específicas de la materia prima empleada en la fabricación del montante de la hoz: un bastón o vara de origen vegetal apropiada que presentaría una disminución progresiva de la anchura desde su base (o parte por la que se produjo el arranque o seccionado del tronco o ramal del árbol, cualquiera que sea el caso) hacia el otro extremo; esto es aún perceptible pese al estado actual del mango y a la posible regularización que pudo afectarle, donde el fragmento a representa claramente la porción más amplia. A su vez dentro del propio fragmento a el extremo más ancho corresponde a la parte que hemos indicado ofrece evidentes visos de fractura. En segundo lugar, estaría la reconstrucción original efectuada por Ponsell (1926, lám. VI-A) -ver fig. $3, n .{ }^{\circ} 3-$. Este autor asistió personalmente al descubrimiento de los restos de la hoz (concretamente del mango), por lo que su montaje es el que más posibilidades encierra de corresponderse con la real disposición de los fragmentos dentro de la estructura primitiva del útil; todo suponiendo que los restos hubieran permanecido conservando aquella estructura o disposición primitiva. De hecho, el montaje de Ponsell es el que normalmente ha sido utilizado para las distintas representaciones de la hoz. Unicamente difieren de éste los aportados por M. Tarradell (1965, foto $\left.n .^{\circ} 11\right)$-ver fig. 3, n. ${ }^{\circ} 1-$ y por J. Aparicio (1976, fig. 13, A). En ambos autores (el montaje que ofrecen es idéntico), el cambio radica no en la posición específica de los fragmentos, sino en su exacta orientación, en la cual se ven afectados los fragmentos $a$ y $b$. Para el caso del fragmento $b$, esto no constituye ningún obstáculo para la cuestión que estamos debatiendo; sí en cambio por lo que respecta al fragmento $a$. Como señalábamos en su epígrafe correspondiente, este fragmento presenta uno de los extremos (concretamente el más estrecho) aparentemente regularizado por un corte seccional; creemos que este carácter es el que probablemente debió sugerir a Tarradell y Aparicio la funcionalidad como empuñadura - por un lado- de dicha porción, y - por otro- el cambio de orientación de la misma según la dada por Ponsell. Independientemente de la intencionalidad del aserrado, que a falta de noticias confirmadoras bien pudiera tratarse de un seccionado antiguo para la obtención de una muestra con la que poder averiguar la naturaleza del soporte, y aun suponiendo que fuera ciertamente una regularización del extremo proximal de la empuñadura, todavía restaría la cuestión de la ranura. El que ésta sea visible en toda la longitud del fragmento ya invalidaría su carácter de asidero. Respecto a su exacta orientación, permanecen los razonamientos expuestos con anterioridad, y en los que las indicaciones de Ponsell nos parece lo más sugerente.

De toda esta discusión, extraeríamos los puntos siguientes:

- que el fragmento $a$ es - a todas luces- el más proximal de los fragmentos conservados, dentro de la estructura general del mango de la hoz.

- que su parte más ancha parece corresponder, a su vez, a su extremo más proximal.

- que este extremo se encuentra perceptiblemente fracturado.

- que la ranura de fijación es reconocible en toda la extensión del fragmento donde se localiza.

Visto lo cual, concluiríamos que los fragmentos de la hoz de Menente que en la actualidad subsisten no corresponden a una pieza entera, sino que se trata de los restos incompletos de la hoz original. En realidad, y siendo más precisos, cabría hablar de los restos incompletos de un mango de hoz.

Otro problema particular a resolver sería el de las dimensiones que pudiera tener la hoz entera y el número de armaduras integrantes.

En cuanto a las armaduras, en las diversas representaciones de la hoz aparece un número variante de ellas, según los autores; precisión ésta que no debe concordar con la realidad por las razones antes apuntadas, máxime cuando la mayoría de veces se trata de reconstrucciones circunstanciales. Es el caso de la realizada por Ponsell (fig. $3, n .^{\circ} 3$ ), donde solamente se observan 3 armaduras; en los otros casos, el número de éstas asignado a la hoz es generalmente de 11 (ver como ejemplo la reproducción de Tarradell, fig. 3, n. $^{\circ} 1$, en este mismo texto). Solamente difiere de los montajes corrientes el de Childe (fig. 3, n. ${ }^{\circ}$ 2), en el cual nos detendremos por lo original de su versión. La reconstrucción de este autor está bastante razonada, tanto por lo que 
concierne al número de armaduras como a la morfología general del mango. En lo tocante a las primeras, Childe enmangaba distalmente 6 dientes, tal vez basándose en que éste era el número de armaduras que aparecía en el mismo departamento que los restos del mango, y quizás guiándose de 10 escrito por Pericot y Ponsell de que se encontraron «junto a los restos de hoz de madera» (PERICOT Y PONSELL, 1929: 6), cosa que no es del todo cierta y sobre la que nos hemos extendido en otro epígrafe. Por lo que se refiere al mango, Childe figuraba proximalmente una serie de acomodamientos destinados a la fijación de los dedos en el momento de empuñar la pieza. Estos ajustes anatómicos no son tales, sino los vaciados producidos por la alteración y pérdida de materia del soporte, que no tienen la regularidad morfológica y disposicional que les confiere Childe. Por lo demás, no es necesario repetir los puntos que acabarían de poner de manifiesto las dudas inherentes a la reconstrucción propuesta por este autor.

Dada la ausencia de paralelos al caso de Menente a los que acudir en el propio marco peninsular y para el mismo período cultural, las dificultades para establecer el número de armaduras y las dimensiones primitivas de la hoz estudiada son, pues, bien notorias. Sin embargo, el hallazgo de un conjunto de dientes de hoz (17 piezas) que se encontraron íntimamente asociados en una zona precisa de uno de los sectores de excavación del poblado del Bronce Valenciano de la «Muntanya Assolada» (Alzira, Valencia), campaña de 1985 bajo la dirección de B. Martí, puede ayudarnos a resolver algunas de las cuestiones planteadas. En principio, las circunstancias señaladas del hallazgo permiten suponer, aunque no se encontraron los restos del montante, que bien pudiera tratarse de un grupo de armaduras correspondientes todas ellas a una única hoz. Partiendo de esta hipótesis, y por el hecho de las concordancias tanto al nivel de la tecnomorfología y tipometría de los dientes de hoz como del marco cronológico-cultural de ambos yacimientos, el caso de la Muntanya Assolada podría muy bien ser trasladado, con todas las lógicas reservas, al del Mas de Menente, proporcionando en este sentido, y por lo que aquí nos ocupa en especial, una primera indicación sobre el número de armaduras que pudiera corresponder a la hoz del segundo yácimiento. Esto, asimismo, nos permitiría especular sobre las dimensiones generales del útil en consideración.
Así, si utilizamos el número de 17 armaduras como probable para la hoz de Menente, y si tenemos en cuenta la longitud media de éstas anteriormente cifrada $(2,30 \mathrm{~cm}$.), resultaría un largo para la ranura de $39,10 \mathrm{~cm}$. Para obtener las dimensiones totales del mango haría falta aún, por una parte, añadir al valor de la ranura el de la distancia que la supera del apéndice terminal de la hoz, don-

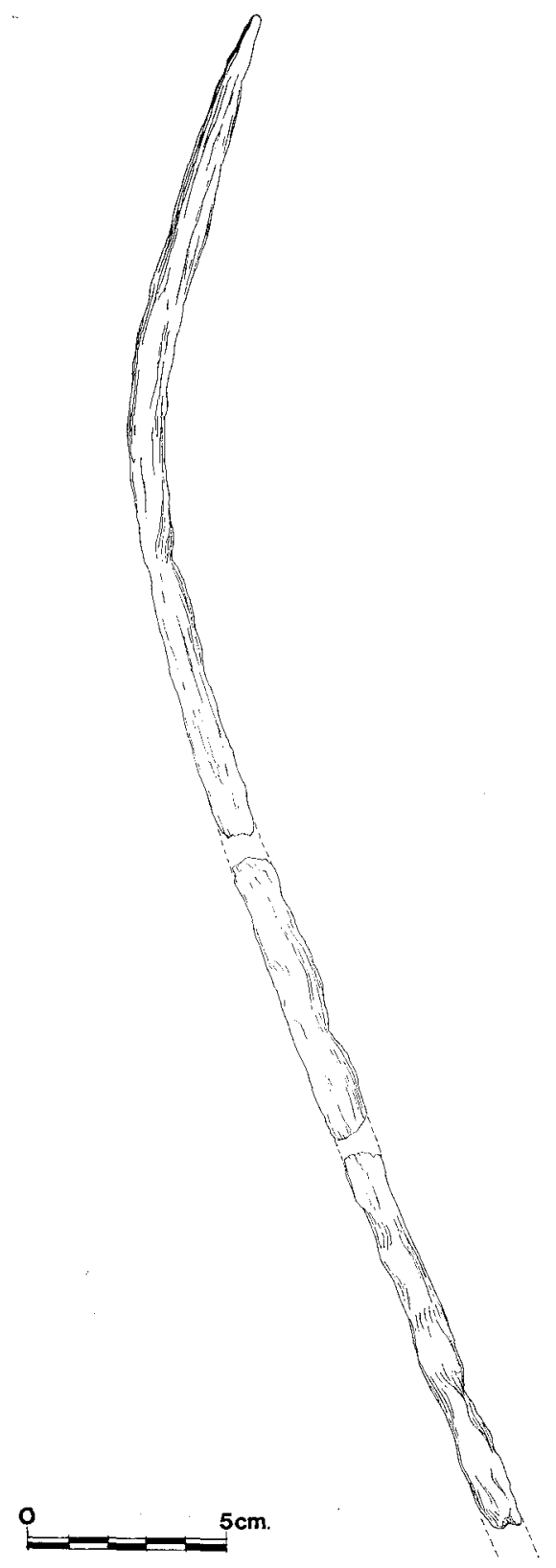

Fig. 5.-Disposición de los fragmentos en el mango de la hoz de Menente. (Dibujo de F. Chiner). 
de ésta se interrumpe distalmente y que es claramente observable en el fragmento $c$, que sería de $2,5 \mathrm{~cm}$.; por otra parte, tendríamos que sumar la extensión presumible de la empuñadura, a la que podemos dar un margen razonable de 10 ó $12 \mathrm{~cm}$. Todas estas mediciones apuntan hacia una longi-

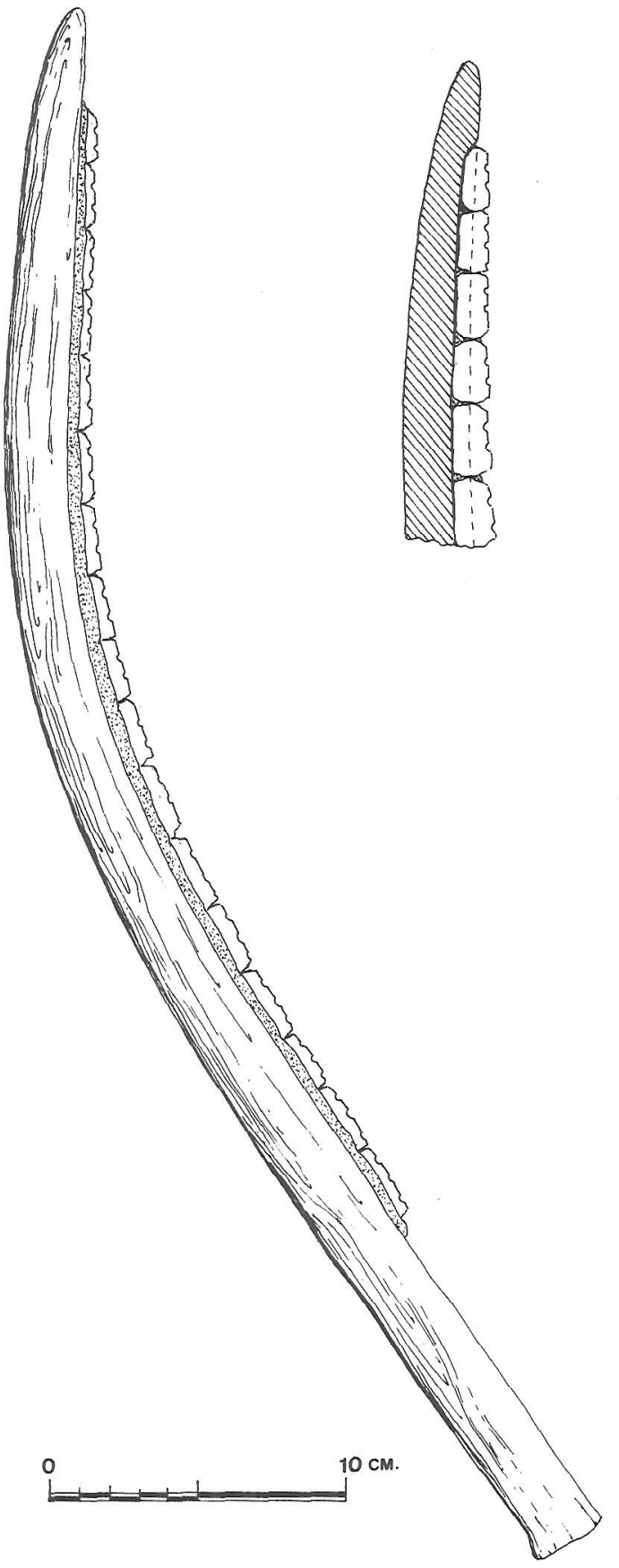

Fig. 6.-Reconstrucción hipotética de la hoz de Menente. (Dibujo de $\mathrm{F}$. Chiner). tud del montante de $51,60 / 53,60 \mathrm{~cm}$., por lo que no es descabellado suponer que el largo total de la hoz de Menente podría estar comprendido entre 45 y $55 \mathrm{~cm}$.

Del mismo modo, fijaríamos una anchura media para la hoz ligeramente superior a los $2 \mathrm{~cm}$. Nos hemos basado para ello en la medida de anchura que presenta el mango en su estado actual $(1,25 \mathrm{~cm}$.) - recordando que, a causa de la degradación, se encuentra esta anchura casi al mismo nivel de lo que sería el fondo de la ranura de fijación-, a la que se ha añadido la mitad de la media de anchura de los dientes de hoz $(0,80 \mathrm{~cm}$.), extensión máxima o poco más que cabe suponer iría inserta en el montante para dejar sobresalir, a su vez, una parte mínima funcionalmente útil. Indirectamente, estas valoraciones permiten aproximarnos con cierto grado de certeza y teniendo en cuenta la escala a que están realizadas, a la propia

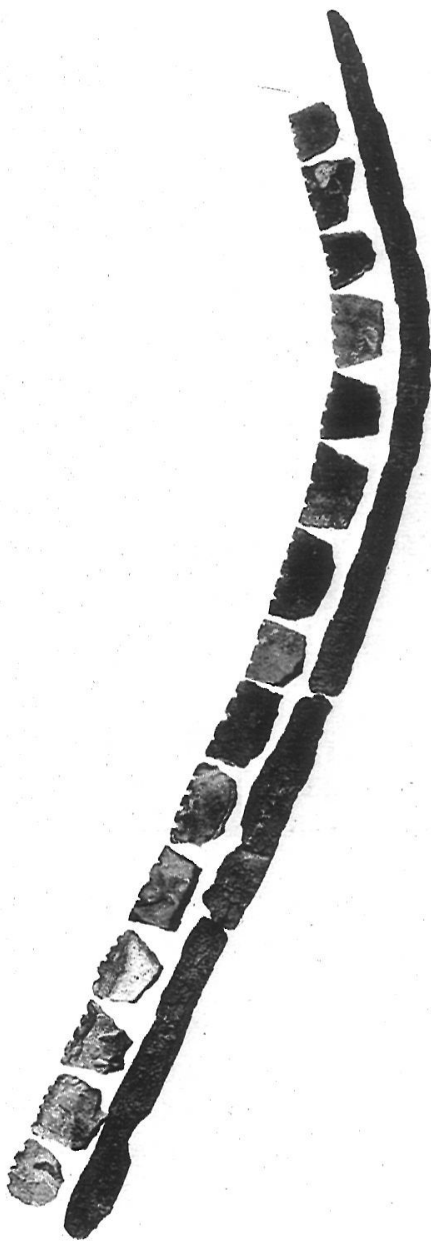

Lám. II.-Montaje actual de la hoz de Menente. 
profundidad de la ranura en el mango, que evidentemente habría de estar centrada alrededor de los $8 \mathrm{~mm}$.

Quedaría por entrever el modo de fijación de las armaduras en la hoz. Sobre este particular, ni el mango ni los dientes examinados ofrecen restos de materia adhesiva alguna que, cabe suponer, permitiría la sujeción. Pero es probable que se empleara cualquier tipo de resina, mezclada o no con otros productos, de las que hay documentadas y experimentadas para esta clase de herramientas en la propia cuenca mediterránea (CAMPS-FABRER Y COURTIN, 1982-83: 19).

Por último, las consideraciones y datos expuestos nos han permitido intentar una reconstrucción hipotética de la hoz de Menente como la que ofrecemos en la fig. 6 .

\section{CONTEXTO TECNOLÓGICO Y CULTURAL}

En el capitulo anterior nos hemos encontrado en la tecnología especializada de la hoz de Menente; en éste, veremos la caracterización de la pieza en el marco de una tecnología general de las hoces prehistóricas $\mathrm{y}$, asimismo, valoraremos su exacta significación cronológico-cultural; esto último teniendo en cuenta el preciso ámbito geográfico que afecta al caso de Menente, pues la extrapolación de datos de una a otras áreas de desarrollo cultural comporta, como observamos, serios inconvenientes en todo estudio de tecnología prehistórica. De todos modos, en cualquier discusión de esta índole siempre es forzado recurrir a ejemplos externos que permitan dar consistencia a los argumentos empleados.

En términos de una clasificación tecnológica general, la hoz de Menente es un claro exponente del tipo de hoz compuesta (COURTIN Y ERROUX, 1974). Estas hoces estarían caracterizadas -obviamente - por un montante o armazón ranurado, de diversa naturaleza y morfología, al cual se adherirían, con ayuda a cualquier materia colante, una serie de armaduras de sílex también de variada morfología. En el caso que nos concierne, $\mathrm{y}$ hemos visto que se trata de un mango de madera marcadamente curvo y de los característicos «dientes de hoz» como armaduras integrantes.

Según un reciente ensayo de clasificación de las hoces prehistóricas en base a la forma del montante y al modo de enmangue y características de las armaduras, debido a D. Helmer (1983), la pieza de Menente podría adscribirse de entrada al tipo III $d$ de este autor: hoces con cuerpo curvo y enmangue paralelo al eje del mismo de varios elementos retocados; los ejemplos ilustrativos de este tipo serían la hoz de Solferino (Italia) y la de Kahun (Egipto) (HELMER, 1983, fig. III; Pl. II, n. ${ }^{\circ} 1$ y 2) -ver fig. $7, n .^{\circ} 1$ y 2 - Sin embargo, en su morfología general y en el tipo de armaduras que conlleva, la hoz de Menente se aparta notablemente de aquéllas, acercándose por el tipo de soporte - aunque no de las armaduras y su disposición- a los ejemplares de Karanovo (Bulgaria) (ibid., fig. III) o de Hacilar (Turquía) (ibid., fig. III; Pl. III, n. ${ }^{\circ}$ ), tipos III $c$ y IV $d$ de Helmer, respectivamente - ver fig. $7, \mathrm{n} .^{\circ} 3$ y $4-$. Tal vez por esto habría que reconsiderar la antigua clasificación de Monteagudo, en que la hoz de Menente constituía un tipo particular (MONTEAGUDO, 1956, fig. 1, n. ${ }^{\circ} 13$ ), trabajo que, por otra parte, ha pasado totalmente desapercibido en las síntesis más recientes.

A la vista de esto, son del todo constatables las limitaciones que comporta cualquier intento de clasificación efectiva de las hoces prehistóricas, y sobre todo el de hacer cuadrar en ellas todos los tipos de hoces existentes. Además, otro problema que subyace en el tema, y en el cual coinciden todos los autores, es el de la inviabilidad de una clasificación tecnológica para las hoces de carácter evolutivo, y mucho más en que el modelo tenga una aplicación general; de ahí que los esquemas usuales sean totalmente atemporales y que estén realizados desde un plano puramente descriptivo.

Esto no quiere decir que en la práctica y en una zona determinada, no pueda discernirse un cierto grado de evolución técnica (aunque mejor cabría hablar, más que de evolución, de «variación» tecnológica), pero normalmente suele ser específica a esa zona (con límites más o menos extensos). Esta consideración se ve con mejor claridad al analizar el caso de las armaduras de hoz. Ante la general ausencia de los soportes, estas piezas son normalmente los únicos testimonios con que solemos contar para inferir las características formales de la hoz entera. Esto puede llevarnos en principio a un conocimiento de las técnicas empleadas por un grupo particular en un área y un momento determinado para la fabricación de sus hoces.

Traducido esto - si es posible- a un plano secuencial, debe ser probable asimismo discernir, mediante la constatación de las variaciones morfo- 
técnicas de las armaduras y las lógicas operaciones de inferencia en el sentido apuntado, la particular evolución tecnológica de las hoces en un área concreta. A otro nivel estaría la cuestión de la convergencia o difusión de las técnicas, que habría que considerar convenientemente en un marco de discusión general.

Esta perspectiva de regionalización es de gran interés para el caso que nos ocupa, pues la tecnología de la hoz de Menente sólo puede ser valorada, justamente y en aquel sentido, en el preciso contexto geográfico-cultứral que directamente le afecta, esto es, la vertiente mediterránea peninsular y, más en concreto, su zona central.

El tema de las hoces peninsulares, si exceptuamos la síntesis que hizo en su día Monteagudo referida al conjunto de estas herramientas repartidas en el ámbito europeo, y donde se dedicaba un concreto apartado con detalle de los casos concernientes a esta área (MONTEAGUDO, 1956), no ha sido tratado con demasiado detenimiento. Bastante anteriormente a Monteagudo, habría que hacer mención de otro trabajo importante para la época, debido a A. Vayson de Pradenne, en el cual, y a propósito de la valoración comparativa de la hoz de Solferino, se pasaba revista a las hoces conocidas deteniéndose asimismo en los ejemplares ibéri-

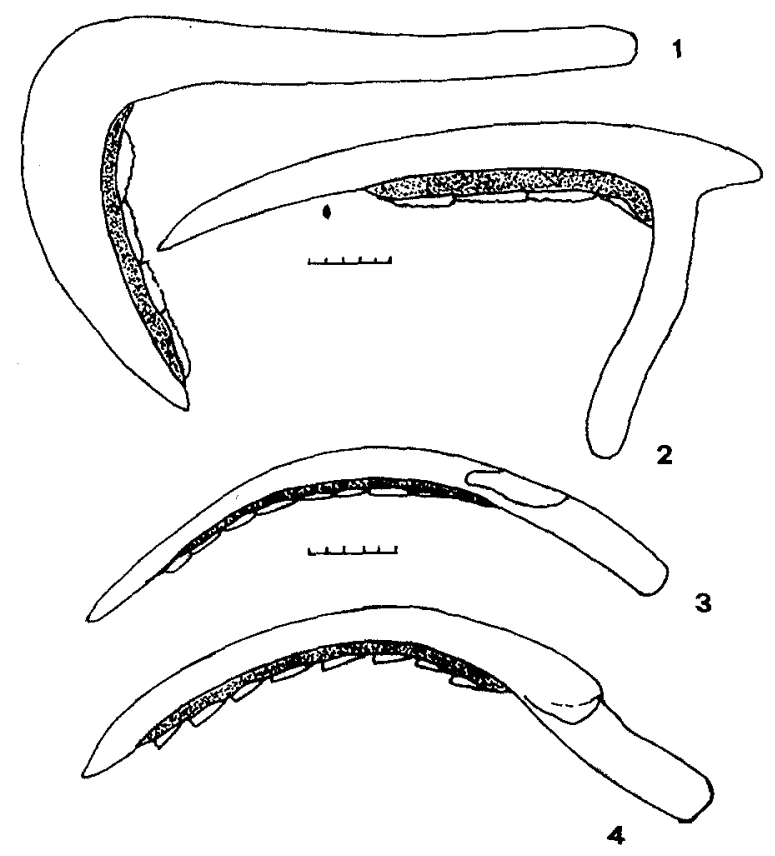

Fig. 7.-Algunos tipos de hoz según reconstrucción de D. Helmer (1983): N. ${ }^{\circ} 1$, Solferino; $n .^{\circ} 2$, Kahun; $n .^{\circ} 3$, Hacilar; n. ${ }^{\circ} 4$, Karanovo. cos (VAYSON DE PRADENNE, 1918-19). Ya más reciente, y tocando de pasada el caso peninsular, es la también señalada síntesis sobre las hoces de la cuencia mediterránea de Camps-Fabrer y Courtin (1982-83). Esto en cuanto a trabajos de carácter general. Fuera de ellos, podemos encontrar algunas precisiones sobre el tema en algunos estudios dedicados a un yacimiento o a un período en concreto; reseñaríamos, por lo que aquí nos afecta y casi por su exclusividad, aquellos que han estado centrados en las hoces neolíticas de la zona que nos ocupa y, más directamente, en sus armaduras, con motivo de análsis más generales de la industria lítica de la etapa (JUAN-CABANILLES, 1984; MARTÍ Y JUAN-CABANILLES, 1984; FORTEA, MARTÍ Y JUAN-CABANILLES, 1985). Por lo demás, hay referencias puntuales sobre las armaduras de hoz, con más o menos detalle, en casi todas las publicaciones que han tratado materiales y yacimientos desde el Neolítico.

Evidentemente, la falta de estudios precisos, sumada al desconocimiento que se tiene en líneas generales de la industria lítica de determinadas etapas importantes, hace que un estudio evolutivo de las hoces en nuestro ámbito de trabajo adquiera un carácter totalmente provisional.

Las primeras hoces de que se tiene noticia en la fachada mediterránea ibérica, especialmente en su zona central, corresponden al Neolítico antiguo de cerámicas cardiales, momento en que se inicia la economía cerealística en este marco. Nos basaremos para su singularización en los recientes estudios de industria lítica de que han sido objeto dos de los yacimientos más representativos de la fase, la Cova de l'Or (Beniarrés, Alacant) y la Cova de la Sarsa (Bocairent, València), trabajos ya citados anteriormente. El análisis de la industria lítica de estos yacimientos ha permitido apreciar la gran significación de las armaduras de hoz (definidas por el lustre o pátina brillante que presentan) en el contexto tecnológico y funcional del Neolítico antiguo. Por su parte, las características de las hoces han sido inferidas a partir de la valoración de estas armaduras, único documento que ha prevalecido de los útiles completos.

Sintéticamente, las armaduras del Neolítico cardial, designadas como elementos de hoz y, como ya hemos visto en otro epígrafe, claramente distanciados semántica y culturalmente de los «dientes de hoz» por Martí (1977), se caracterizan por estar elaborados siempre sobre productos la- 
minares, con el sílex como materia prima exclusiva. En general, son pocas las modificaciones que han sufrido los soportes primarios, conservando los caracteres propios de la talla. La mayoría de veces la modificación se traduce solamente en una fracturación intencional de uno o ambos extremos, a fin de conseguir, pai ace ser, series dimensionalmente estandars de piezas intercambiables entre sí. Cuando pueden observarse acomodamientos, se trata de alguna truncadura (recta $\mathrm{u}$ oblícua) para los lados cortos o extremos (fig. 8, n. ${ }^{\circ} 4$ y 7 ); de algún retoque contínuo o parcial, casi nunca abatiendo el borde, para los dorsos (fig. $8, \mathrm{n} .^{\circ} 6$ ), además de alguna escotadura o adelgazamiento lateral, localizados proximal o distalmente (fig. $8, \mathrm{n}^{\circ}$ 5 ), para estos mismos. Los filos suelen conservar el borde bruto de talla, únicamente afectado por algunos retoques o descamaciones claramente debidos al uso; en ocasiones el filo puede presentar también algún acomodamiento del tipo de la escotadura, siempre terminal. El lustre, bien visible en todas estas armaduras, se dispone siempre oblícuamente con respecto al eje de la pieza. Este carácter, junto con el resto de adecuaciones tecnológicas señaladas, abogan por un ensamblaje oblícuo de los elementos en el mango (inserción en espiga), conformando un filo dentado en que cada armadura constituye un único diente.

A falta de un análisis tecnológico más centrado que permita conocer con detalle las características morfológicas del mango, número de armaduras, dimensiones generales, etc., $y$ en el cual estamos inmersos en el momento presente, la reconstrucción hipotética de las hoces del Neolítico antiguo de Or y Sarsa podría ser la ofrecida por Martí (1983, fig. 15) -ver fig. 9 , n. ${ }^{\circ} 1-$, hoces con un montante curvo guarnecido con elementos escalonados, recordando al modelo de Karanovo (fig. 7, n. $\left.{ }^{\circ} 4\right)$. Este tipo de hoz es el que ha debido perdurar durante el transcurso del Neolítico, sin cambios substanciales.

En cuanto al Eneolítico, el conocimiento que se tiene de las hoces es bastante precario, ligado a la misma circunstancia que rodea a la industria lítica del período. Si bien se tiene documentación a nivel descriptivo de los materiales exuberantes de los enterramientos colectivos en cueva (puntas de flecha, grandes hojas, etc.), poco es lo que se sabe de la tecnología de base que ampara esa producción, y menos de la exacta significación en términos de funcionalidad de algunos de sus elementos. Así, calificar meramente de «rituales» las grandes hojas de los ajuares mortuorios carece de sentido cuando, en la mayoría de ocasiones, son visibles en ellas las trazas de usos evidentes. A todo esto contribuye claramente la fragmentaria información que poseemos de los asentamientos estables, a lo que se une las deficiencias en el estudio y publicación de materiales (por lo que nos concierne líticos) en los casos mejor conocidos. Ciertamente, la clave para el conocimiento de la industria lítica del Eneolítico se encuentra en los poblados y no en las cuevas sepulcrales, hasta ahora casi únicas proveedoras de información en este sentido.

Con todo, la vista de los materiales del poblado de la Ereta del Pedregal (Navarrés, València), yacimiento representativo de la etapa y actualmente en proceso de revisión, nos permite un primer acercamiento a las posibles armaduras y, con ello, a las características generales de las hoces eneolíticas. Por lo que hemos podido observar, las armaduras de la Ereta se caracterizan por su elaboración sobre

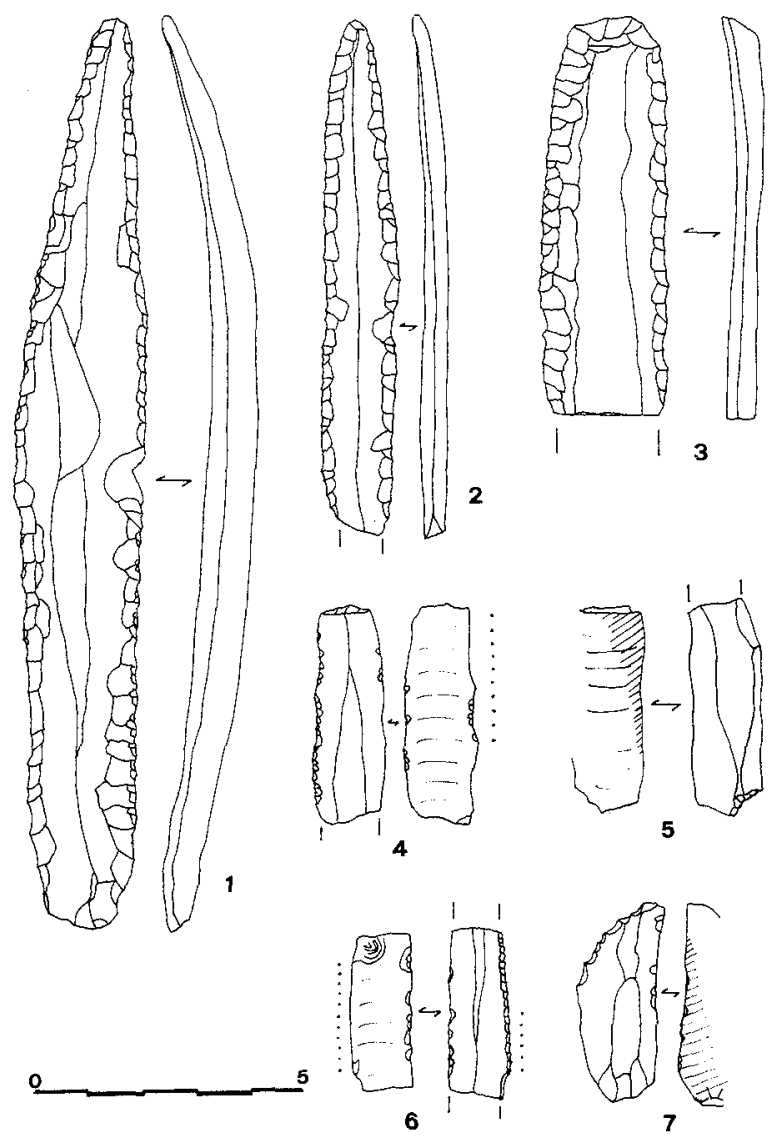

Fig. 8.-Elementos y armaduras de hoz neolíticos y eneolíticos: N. ${ }^{\circ} 1$ a 3, Ereta del Pedregal; n. ${ }^{\circ} 4$ a 7 , Cova de l'Or. 
soportes laminares, de tamaños respetables, en líneas generales mayores que los elementos de hoz neolíticos. Presentan normalmente los dos bordes retocados, con retoques simples o de tendencia plana, contínuos, a veces invasores, obtenidos probablemente por presión (fig. $8, n .^{\circ} 1$ a 3 ). En ocasiones pueden ofrecer algún acomodamiento terminal, que recuerda a un frente de raspador (fig. 8, n. ${ }^{\circ} 3$ ), pero que su finalidad debe ser la misma que la indicada para las truncaduras: favorecer el ensamblaje de las piezas entre sí en el mango de la hoz. El lustre, cuando puede observarse, se encuentra siempre en disposición paralela al eje de la pieza, lo que revela un enmangue rectilíneo. Las consideraciones sobre las dimensiones de estas armaduras permiten inferir su adecuación a dos tipos de hoces tecnológicamente distinas. Por una parte, aquellas piezas enteras con longitudes superiores a
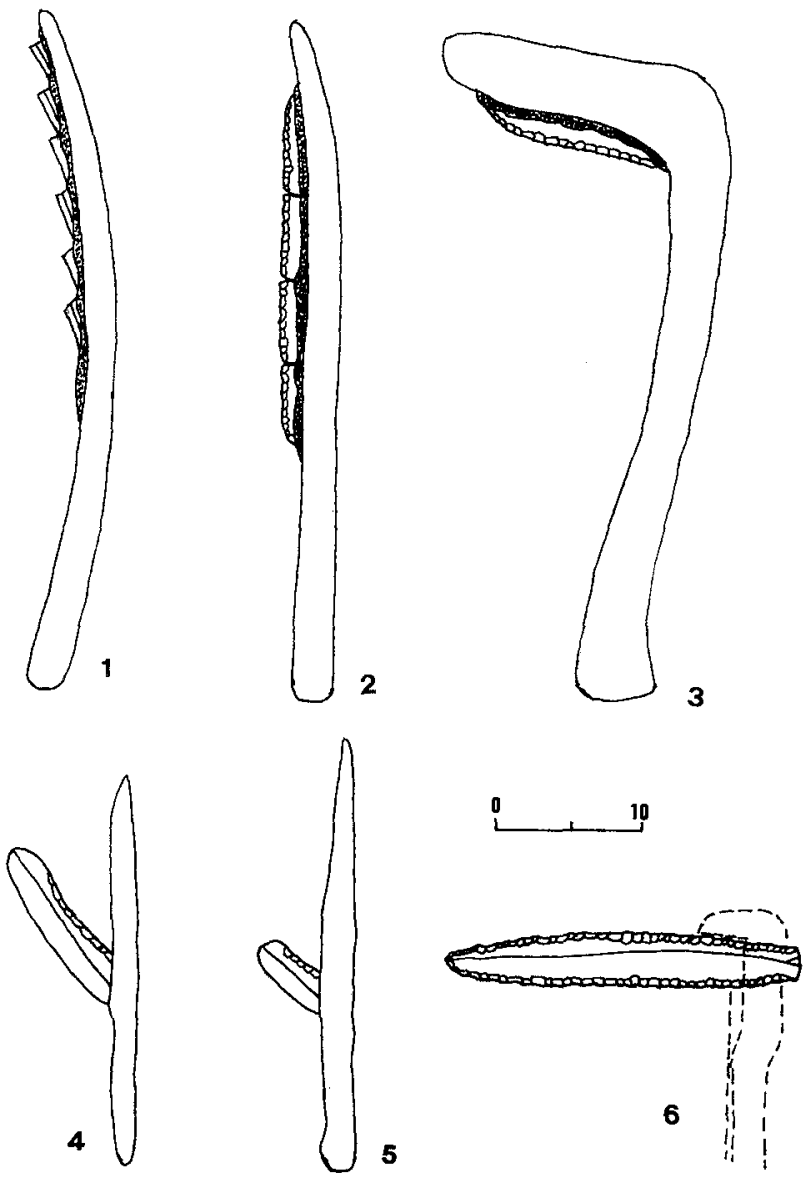

Fig. 9.-Diversos montajes de hoces: N. ${ }^{\circ} 1$, segủn B. Martí (1983); n. ${ }^{\circ} 4$ y 5, tipos de Egolzwill, según J. Courtin y J. Erroux (1974); n. ${ }^{\circ}$ 6, según L. Monteagudo (1956). $\operatorname{los} 15 \mathrm{~cm}$. (fig. $8, \mathrm{n}^{\circ} 1$ ), podrían armar hoces simples, con un único elemento inserto en un mango de madera rectilíneo o poco curvo, paralelamente al eje del mismo; otra forma de inserción que no cabe descartar podría ser con la única armadura dispuesta oblícuamente respecto al mango, éste también rectilíneo, del modo en que aparece documentado en ciertos yacimientos suizos, caso de Egolzwill (COURTIN Y ERROUX, 1974) -ver fig. 9, n. ${ }^{\circ} 4$ y 5-. Asimismo, otras formas de montaje para las grandes hojas podrían ser las indicadas en la fig. 9 ( $n .^{\circ} 3$ y 6 ). Por otra parte, las piezas con fracturas o acomodamientos terminales, de dimensiones más pequeñas, podrían ser constitutivas de hoces compuestas; el armazón en este caso puede ser de variada morfología, aunque lo suponemos rectilíneo y con una serie determinada de armaduras dispuestas paralelamente a su eje, delineando un filo contínuo (fig. $9, \mathrm{n} \cdot{ }^{\circ} 2$ ).

La coexistencia de estos dos tipos de hoces (simples y compuestas), y a falta de información directa, puede presuponerse ya desde el Neolítico final, aunque la distinción habría que hacerla al nivel de la morfología de las armaduras y de sus modos de enmangue con respecto a los casos eneolíticos. Esto vendría confirmado por las referencias que tenemos de otras áreas limítrofes, en particular del Sudeste francés, donde la hoz simple, ligada a grandes hojas apenas retocadas y con lustre, puede arrancar desde el Chassense (Neolítico medio), y estando muy bien reconocida en el Neolítico final y el Calcolítico de la zona, en esta última fase con piezas similares cuidadosamente retocadas del tipo que hemos señalado para la Ereta (CAMPSFABRER Y COURTIN, 1982-83).

El tipo de hoz para la Edad del Bronce ya lo hemos visto, viniendo definido en sus características técnicas generales y clase de armaduras por el ejemplar de Menente.

Aunque esta modalidad de hoz se encuentra bien individualizada para la etapa, pareciéndole ser específica dada la exclusividad y gran repartición de sus armaduras o «dientes de hoz» (ENGUIX, 1975; DE PEDRO, 1985), es posible que su origen haya que buscarlo en fases inmediatamente anteriores. A ello parece apuntar el hallazgo de piezas del tipo diente de hoz, asociadas a vasos campaniformes, en algún yacimiento encuadrable por esto en el Eneolítico final u Horizonte Campaniforme de Transición de la zona (BERNABEU, 1984). Fuera de este marco, y a título indicativo 
por lo que puede confirmar este hecho, dientes de hoz han sido asimismo determinados en niveles claramente del Cobre Pleno andaluz del poblado de los Castillejos (Montefrío, Granada) (ARRIBAS Y MOLINA, 1979).

Tras esta somera evaluación de los datos, las características tecnológicas de las hoces y su evolución o variación en la zona oriental de la fachada mediterránea peninsular quedarían así sintetizadas:

- Para el Neolítico:

- Hoces compuestas.

- Mangos indeterminados, probablemente curvos.

- Filos dentados por escalonamiento de elementos simples (inserción oblícua respecto al eje del mango).

- Para el Eneolítico:

- Hoces simples y compuestas.

- Mangos indeterminados.

- Filos rectos, por una o varias armaduras retocadas insertadas paralelamente al eje del mango.

- Para el Bronce:

- Hoces compuestas.

- Mangos curvos.

- Filos delinculados por yuxtaposición de varias armaduras intencionalmente dentadas insertas paralelamente al eje del mango.

Este esquema a una escala muy general, especialmente caracterizadora de cada etapa en concreto, pues las pervivencias de modos o el arranque de nuevos desde etapas intermedias anteriores a su plena individualización, son problemas que habría que dilucidar a partir de estudios más precisos de las constantes tecnológicas e industriales de cada período y fase de transición.

Dentro de esta evolución, el caso de Menente representaría en cierta manera la culminación de todo un proceso de cambios e interacciones tecnológicas, cuyas precisas directrices se nos escapan de momento, y que tocará a su fin con la generalización de las hoces de hierro ya en la etapa posterior de la Cultura Ibérica.

\section{BIBLIOGRAFIA}

APARICIO, J., 1976: Estudio económico y social de la Edad del Bronce Valenciano. Publicaciones del Archivo Muni- cipal de Valencia, estudios monográficos, 8, Valencia, $284 \mathrm{p}$.

ARRIBAS, A. y MOLINA, F., 1979: Nuevas aportaciones al inicio de la metalurgia en la Península Ibérica. El poblado de los Castillejos de Montefrío (Granada). Proceedings of the fifth Atlantic Colloqium «The Origins of Melallurgy in Atlantic Europe», Dublin, pp. 7-32.

BALLESTER, I., 1929: El Servicio de Investigación Prehistórica y su Museo de Prehistoria. Tirada a parte de la Memoria reglamentaria de la Secretaría de la Diputación, correspondiente a 1928, Valencia, pp. 14-16.

BERNABEU, J., 1984: El vaso campaniforme en el País Valenciano. Trabajos Varios del Servicio de Investigación Prehistórica de Valencia, n. ${ }^{\circ} 80$, Valencia, $140 \mathrm{p}$.

CAMPS-FABRER, H. y COURTIN, J., 1982-83: Essai d'approche technologique des faucilles préhistoriques dans le bassin mediterranéen. Travaux du Laboratoire d'Anthropologie, Aix-en-Provence, $26 \mathrm{p}$.

COURTIN, J. y ERROUX, J. 1974: Aperçu sur l'agriculture préhistorique dans le Sud-Est de la France. Bulletin de la Société Préhistorique Française, t. 71, París, pp. 321334.

CURWEN, E. C., 1930: Prehistoric flint sickles. Antiquity, t. 4, Gloucester, pp. 179-186.

CHILDE, V. G. $(\mathrm{s} / \mathrm{r})$ : The balanced sickle. Aspects of $\mathrm{Ar}-$ chaeology in Britain and beyond, pp. 39-48. El artículo este lo hemos consultado en la Biblioteca del S.I.P., que se encuentra archivado en forma de separata sin más referencias que las expuestas. Debe ser anterior al año 1951, pues ésta es la fecha de ingreso en dicha biblioteca.

DE PEDRO, M. J., 1985: La industria lítica de la Mola de Agres, Papeles del Laboratorio de Arqueología de Valencia-Saguntum, 19, Valencia pp. 85-106.

ENGUIX, R., 1975: Notas sobre economía del Bronce Valenciano. Papeles del Laboratorio de Arqueología de Valencia, Valencia, pp. 141-158.

- 1980: La Edad del Bronce. En Nuestra Historia, t. I, Mas Ivars Editores, Valencia, pp. 151-170.

FLETCHER, D., 1974: Museo de Prehistoria de la Diputación Provincial de Valencia. Publicaciones del Círculo de Bellas Artes, Valencia, 209 p.

FORTEA, J., 1973: Los complejos microlaminares y geométricos del Epipaleolítico mediterráneo español. Memorias del Seminario de Prehistoria y Arqueologia, n. ${ }^{\circ} 4$, Salamanca, $545 \mathrm{p}$.

FORTEA J.; MARTÍ B. y JUAN-CABANILLES, J., 1985: L'industrie lithique du Néolighique ancien dans le versant mediterranéen de la Peninsule Ibérique. Comunicación al Symposium «Chipped Stone Industries of the Early Farming Cultures in Europe», Krakowie, octubre 1985. (En prensa).

G.E.R.V., 1973: Gran Enciclopedia de la Región Valenciana, t. IV, GAI-IND, Voz «hoz», p. 261.

GÓNGORA, M., 1868: Antigüedades prehistóricas de Andalucía. Madrid, $158 \mathrm{p}$.

HELMER, D., 1983: Les faucilles et les gestes de la moisson. Travaux de la Maison de l'Orient, n. ${ }^{\circ} 5$, Lyon, pp. 189 198.

JUAN-CABANILLES, J., 1984: El utillaje neolítico en sílex del litoral mediterráneo peninsular. Papeles del Laboratorio de Arqueología de Valencia-Saguntum, 18, Valencia, pp. 49-102. 
MARTÍ, B., 1977: Cova de l'Or (Beniarrés, Alicante). Vol. I. Trabajos Varios del S.I.P., n. ${ }^{\circ}$ 51, Valencia, 92 p.

- 1983: El naixement de l'agricultura en el País Valencià. Universitat de Valencia, Secretariat de publicacions, València, $132 \mathrm{p}$.

MARTí, B. y JUAN-CABANILLES, J., 1984: Industrie lithique et Neolithique ancien dans le versant mediterranéen de la Peninsule Iberique. Bulletin de la Société Méridionale de Spéléologie et de Préhistoire, $\mathrm{t}$. XXIV, Toulouse, pp. 49-63.

MONTEAGUDO, L., 1956: Hoces de sílex prehistóricas. $R e^{-}$ vista de Archivos, Bibliotecas y Museos, t. LXII, fasc. 2, Madrid, pp. 457-534.
PERICOT, L. y PONSELL, F., 1929: El poblado de «Mas de Menente» (Alcoy, Alicante). Archivo de Prehistoria Valenciana, 1, (1928), Valencia, pp. 101-112.

PONSELL, F., 1926: Excavaciones en la finca «Mas de Menente», término de Alcoy (Alicante). Memorias de la Junta Superior de Excavaciones y Antigüedades, 78, Madrid, 8 p.

TARRADELL, M., 1965: Prehistòria i Antiguitat. En «Història del País Valencià, vol. I, Edicions 62, Barcelona, pp. 19-206.

VAYSON DE PRADENNE, A., 1918-19: Faucille préhistorique de Solferino. Etude comparative. L'Anthropologie, t. 29, París, pp. 393, 433. 